\title{
Zur Theorie der elektrochemischen Darstellung von Plumbichlorid
}

\author{
Von \\ Robert Kremann und Hermann Breymesser \\ Aus dem Chemischen Institut der Universität Graz \\ (Mit 2 Textfiguren)
}

(Vorgelegt in der Suzung am 1. Dezember 1916)

Eine Lösung von Plumbichlorid $\mathrm{PbCl}_{4}$ in Salzsäure, also eine Lösung von Plumbichlorwasserstoffsäure, kann u. a. auf rein chemischem Wege nach Friedrich ${ }^{1}$ durch Einleiten von Chlor in Salzsäure, in der Plumbochlorid $\mathrm{PbCl}_{2}$ suspendiert ist, die also an $\mathrm{PbCl}_{2}$ gesättigt ist, erhalten werden. Auf elektrochemischem Wege haben Elbs und Nübling ${ }^{2}$ eine Lösung von Plumbichlorwasserstoffsäure mit 70 bis $80 \%$ Ausbeute unter folgenden Bedingungen erhalten:

In einem Glastrog, der die Anodenflüssigkeit,. $\mathrm{HCl}$ vom spezifischen Gewicht $1 \cdot 18$, enthält, tauchte ein Diaphragma mit der Kathodenflüssigkeit ( $\mathrm{HCl}$ vom spezifischen Gewicht 1.1) und einer Bleikathode. Die Anode besteht aus zwei dem Diaphragma parallel senkrecht in die Anodenflüssigkeit tauchenden, zickzackförmig gebogenen Bleistreifen einerseits und einer am Boden des Glastroges parallel $\mathrm{zu}$ diesem und senkrecht $\mathrm{zu}$ den Bleielektroden gestellten plattenförmigen Kohlenelektrode andrerseits. In dieser geteilten Anode, in der die Stromverteilung so bemessen ist, daß durch die beiden Bleianoden zusammen

1 Ber., 26, 1434, 1893.

2 Zeitschr. für Elektrochemie, 9, 776, 1903. 
und die Kohlenanode je gleich viel Strom hindurchgeht, wirken die Bleiteile der Anode als Lösungsanoden, indem sie Plumbichlorid bilden, das also die Anodenflüssigkeit jeweilig an diesem Stoff sättigt und in ihr suspendiert wird, die Kohlenteile als unangreifbare. Elektrode, an der Chlor entsteht, das aber in gasförmigem Zustande vornehmlich zu Beginn der Elektrolyse nur in untergeordnetem Maße zur Abscheidung kommt, in der Anodenflüssigkeit also gelöst und weiterhin verbraucht wird. Denn während der Elektrolyse, die unter Eiskühlung durchgeführt wurde, färbt sich die Anodenflüssigkeit orangegelb und es bildet sich eine salzsaure Lösung von Plumbichlorid, aus der sich ebenso wie bei der Herstellung nach Friedrich auf rein chemischem Wege durch Fällung mit geeigneten Salzen, z. B. Ammonchlorid, die entsprechenden Doppelsalze der Plumbichlorwasserstoffsäure fällen lassen. Wenn man den Reaktionsmechanismus der beschriebenen eiektrochemischen Darstellung der salzsauren Lösung von Plumbichlorid betrachtet, so sind zwei Deutungen möglich:

1. Das an der Bleianode sich bildende Plumbichlorid, das sich einerseits in der Salzsäure löst, andrerseits aber in festem Zustande nach abwärts sinkt, wirkt als Depolarisator auf die Chlorabscheidung an der Kohlenelektrode unter Bildung von Plumbichlorid, womit die Bildung dieses Stoffes also durch einen primären Elektrodenvorgang bedingt würde.

2. Es bilden sich primär an den Elektroden nur $\mathrm{PbCl}_{2}$ und $\mathrm{Cl}_{2}$, die dann sekundär in. der Anodenflüssigkeit ganz wie bei der rein chemischen Darstellung nach Friedrich unter Bildung von Plumbichlorid reagieren.

Für die Bildung dieses Stoffes würden. also primäre Elektrodenvorgänge keine wesentliche Rolle spielen.

Zur Prüfung dieser zwei Möglichkeiten haben wir die Zersetzungsspannungen von Salzsäure rom spezifischen Gewicht 1.18 und von Salzsäure vom spezifischen Gewicht $1 \cdot 18$, die jeweils mit $\mathrm{PbCl}_{2}$ gesättigt war, bei $0^{\circ}$ und $21^{\circ}$ an Kohleelektroden verglichen. Die Methode war im Wesen die Ion Le Blanc angegebene. ${ }^{1}$ In zwei weite Glasröhren, die

1 Zeitscht. für phys. Ch., 8, 299. 
mit einer $\mathrm{W}$-förmigen Kapillare verbunden und mit reiner, beziehungsweise mit $\mathrm{PbCl}_{2}$ gesättigter Salzsäure vom spezifischen Gewicht 1.18 beschickt waren, tauchte eine größere Kathode und eine kleine Anode aus Bogenlampenkohle. Letztere war in ihrer Hauptfläche paraffiniert und ließ nur eine kleine wirksame Fläche frei. An die beiden Elektroden wurden nun steigende Spannungen angelegt und je 10 Minuten nach Stromschluß die Ausschläge eines in den Stromkreis zu schaltenden Galvanometers, die also den jeweiligen Stromstärken proportional sind, sowie die zugehörigen Anodenpotentiale gemessen. $Z u$ diesem $Z$ weck war mit dem Anodenschenkel des ob̉en erwähnten Gefäßes durch einen Kapillarhahn eine Wasserstoffnormalelektrode (ein von Wasserstoff béspültes, in 2 norm. Schwefelsäure tauchendes platiniertes Platinblech) verbunden. Setzen wir für diese das Potential Null, so entsprechen die Werte der gemessenen Kette: Anode/Wasserstoffelektrode dem jeweiligen auf die Wasserstoffelektrode bezogenen Anodenpotential. Es wurden hiebei die folgenden zusammengehörigen Werte von Anodenpotential und Galvanometerausschlägen (id est Stromstärken) erhalten:

1. $\mathrm{HCl}$, spezifisches Gewicht 118 , bei $0^{\circ}$ gesättigt mit $\mathrm{PbCl}_{2}$ (Punkte $\times$ in Fig. 1).

$\begin{array}{cc}\text { Anodenpotential in Volt } & \text { Galvanometeráusschlag } \\ 0.605 & 1 \\ 0.669 & 4 \\ 0.735 & 6 \\ 0.814 & 9 \\ 0.915 & 12 \\ 0.956 & 16 \\ 1.016 & 21 \\ 1.051 & 28 \\ 1.090 & 55\end{array}$

2. $\mathrm{HCl}$, spezifisches Gewicht 1.18 bei $0^{\circ}$ (Punkte o in Fig. 1). Anodenpotential in Volt Galvanometerausschlag

$\begin{array}{lr}0.686 & 5 \\ 0.814 & 10 \\ 0.826 & 10 \\ 0.880 & 14 \\ 0.942 & 19 \\ 1.038 & 41 \\ 1.072 & 52\end{array}$


3. $\mathrm{HCl}$, spezifisches Gewicht 1.18 bei $21^{\circ}$ gesättigt an $\mathrm{PbCl}_{2}$ (Punkte $\triangle$ in Fig. 2).

$\begin{array}{cc}\text { Anodenpotential in Volt } & \text { Galvanometerausschlag } \\ 0.663 & 2 \\ 0.715 & 5 \\ 0.741 & 7 \\ 0.787 & 9 \\ 0.825 & 11 \\ 0.838 & 11 \\ 0.896 & 15 \\ 0.956 & 20 \\ 1.020 & 48 \\ 1.053 & 80\end{array}$

4. $\mathrm{HCl}$, spezifisches Gewicht 1.18 bei $21^{\circ}$ (Punkte $\circ$ in Fig. 2).

$\begin{array}{cc}\text { Anodenpotential in Volt } & \text { Galvanometerausschlag } \\ 0 \cdot 710 & 6 \\ 0.802 & 18 \\ 0.880 & 30 \\ 0.890 & 40 \\ 0.938 & 72 \\ 0 \cdot 973 & 109 \\ 0.999 & 148 \\ 1.018 & 196\end{array}$

5. $\mathrm{H}_{2} \mathrm{SO}_{4}, 1$ norm. bei $21^{\circ}$ (Punkte $x$ in Fig. 2).

Anodenpotential in Volt

0.735
$0 \cdot 756$
$0 \cdot 762$
0.882
$1 \cdot 012$
1.097
$1 \cdot 139$
Galvanometerausschlag

$$
\begin{array}{r}
7 \\
9 \\
11 \\
23 \\
37 \\
66
\end{array}
$$$$
102
$$

Trägt "man nun die Abhängigkeit der Anodenpotentiale an der Kohlenanode in Abhängigkeit von den Galvanometerausschlägen graphisch auf, so zeigen bekanntlich die Knickpunkte das Eintreten bestimmter Elektrodenvorgänge an. Betrachten wir die in Fig. 1 und 2 dargestellten, für $0^{\circ}$, beziehungsweise $21^{\circ}$ beobachteten Zersetzungsspannungskurven, 
so sieht man in jeder der beiden auf $\mathrm{HCl}$ vom spezifischen Gewicht $1 \cdot 18$, beziehungsweise auf $\mathrm{HCl}$ vom spezifischen Gewicht $1 \cdot 18$, gesättigt mit $\mathrm{PbCl}_{2}$, bezüglichen Kurven zwei ausgeprägte Kṇickpunkte.

Der erste bei $0^{\circ}$ von 0.83 Volt, bei $21^{\circ}$ bei 0.87 Volt, ist unabhängig davon, ob reine Salzsäure oder mit $\mathrm{PbCl}_{2}$ gesättigte Salzsäure in Verwendung kommt.

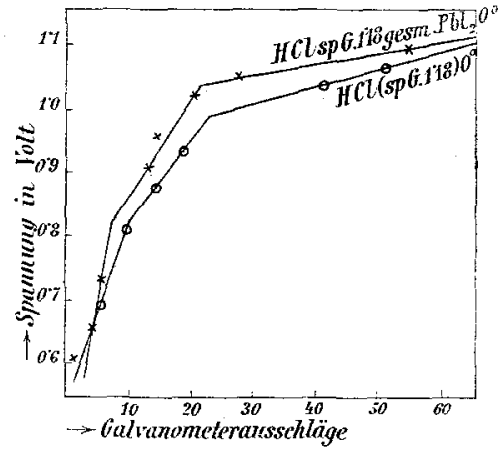

lïg 1.

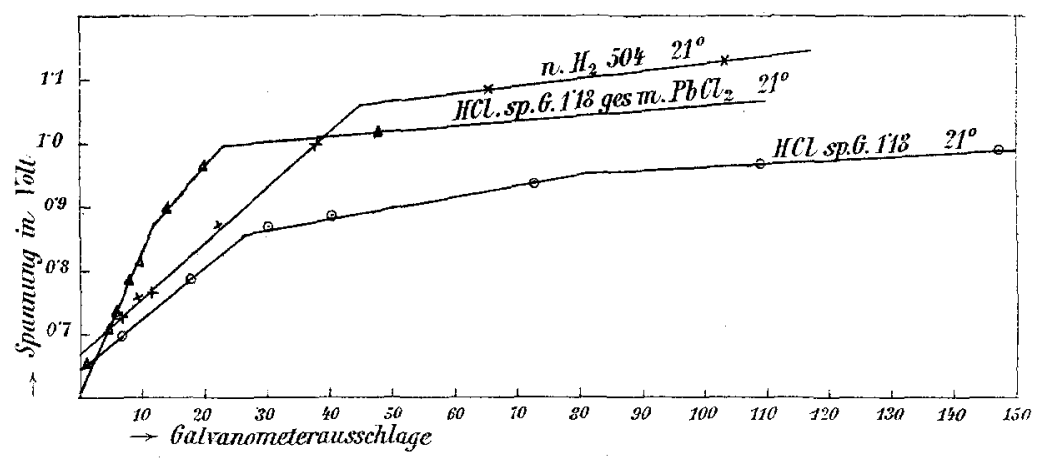

Fig. 2.

Der zweite Knickpunkt liegt für Salzsäure bei $0 \cdot 99$, beziehungsweise 0.95 Volt bei $0^{\circ}$, beziehungsweise $21^{\circ}$, für mit $\mathrm{PbCl}_{2}$ gesättigte Salzsäure bei $1 \cdot 04$, beziehungsweise $1 \cdot 0$ Volt.

Im allgemeinen sind die zweiten Knicke stärker ausgeprägt als die ersten. ${ }^{1}$ Es dürften sich -also erstere auf die Entladung

1 Eine Ausnahme macht nur die auf reine $\mathrm{HCl}$ bei $21^{\circ}$ sich beziehende Kurve. 
der in größerer Menge vorhandenen Ionenart beziehen, aller Wahrscheinlichkeit nach auf die Entladung ron Chlorionen.

Es würde also demgemäß unter den von uns gewählten Bedingungen die Zersetzungsspannung von, Salzsäure an Kohleanoden bei 0.99 , beziehungsweise 0.95 Volt liegen, die bei Sättigung mit $\mathrm{PbCl}_{2}$ um je 0.05 Volt erhöht wurde.

Schon aus diesen Befunden geht als Resultat hervor, daß die Annahme einer depolarisierenden Wirkung ron $\mathrm{PbCl}_{2}$ auf die Chlorabscheidung bei der elektrochemischen Darstellung ron $\mathrm{PbCl}_{4}$ nicht zutrifft, sondern es sich hiebei um eine rein chemische sekundäre Einwirkung zwischen dem auf elektrochemischem Wege gebildeten $\mathrm{PbCl}_{2}$ und $\mathrm{Cl}_{2}$, handelt. Denn andernfalls müßte ja im Gegenteil bei Anweseṇheit ron $\mathrm{PbCl}_{2}$ in Salzsäure eine Erniedrigung der oben erwähnten Zersetzungspunkte eintreten.

Was die Lage der beobachteten Zersetzungspunkte anlangt, so entspricht sie ziemlich gut den an Platin beobachteten Zersetzungsspannungen der Salzsäure, ein Umstand, der uns auch dafür bestimmte, die zweiten Knickpunkte der Chlorionenabscheidung zuzusprechen. Mit steigender Verdünnung nimmt bekanntlich die Zersetzungsspannung der Salzsäure zu, um schließlich den der Sauerstoffabscheidung entsprechenden maximalen Grenzwert zu erreichen. Le Blanc fand die folgenden Zersetzungswerte:

$\begin{array}{lc}\mathrm{HCl}, \text { Mol.in Liter } & \text { Zersetzungspannung in Volt } \\ 2 \cdot 0 & 1 \cdot 26 \\ 1 \cdot 0 & 1 \cdot 31 \\ 0 \cdot 5 & 1 \cdot 34 \\ 0 \cdot 116 & 1 \cdot 41 \\ 0.062 & 1 \cdot 32 \\ 0.031 & 1 \cdot 69\end{array}$

Mit steigender Salzsäurekonzentration dürften die Werte der Zersetzungsspannung noch weiter sinken, wie Messungen von Dolezalek über die elektromotorische Kraft $E$ der Chlorknallgaskette es zeigen.

1 Le Blanc, Lehrbuch der Elektrochemie, 226, II. Atill, 1901. 
Nach Dolezalek ${ }^{1}$ ist für Salzsäure

$\begin{array}{rr}4.98 \text { Mol. in Liter } \quad E=1 \cdot 190 \text { Volt } \\ 6.43 & 1 \cdot 147 \\ 11 \cdot 20 & 1 \cdot 005 \\ 11.62 & 0.999 \\ 12 \cdot 25 & 0.974\end{array}$

Nach dem spezifischen Gewicht der von uns verwendeten Salzsäure ist die Salzsäure zirka 11- bis 12 molar. Die für diese Salzsäure gemessenen zweiter Stärke passen sich unter gleichzeitiger Berücksichtigung des Umstandes, daß wir Kohleanoden verwendet haben, ${ }^{2}$ den Werten der Chlorknallgaskette bei naheliegenden Salzsäurekonzentrationen ziemlich gut an.

Was nun die Erhöhung der Zersetzungsspannung bei Sättigung an $\mathrm{PbCl}_{2}$ anlangt, so läßt sich diese folgendermaßen deuten:

Nach v. Endes ${ }^{1}$ beträgt die Löslichkeit von $\mathrm{PbCl}_{2}$ bei $25.2^{\circ}$ für eine 12.05 molare Säure, wie sie ungefähr unseren Versuchen entspricht, $0 \cdot 1643 \mathrm{Mol}$ i. L. In diesen Lösungen liegt das komplexe Ion $\mathrm{PbCl}_{4}^{\prime \prime}$ vor, d. h. die Konzentration der Chlorionen in starker Salzsäure wird durch gelöstes $\mathrm{PbCl}_{2}$ herabgemindert, wodurch die Abscheidung von Chlor aus solchen Lösungen bei höherer Spannung erfolgt.

Was nun die ersten Knickpunkte bei $0 \cdot 83$, beziehungsweise $0.87 \mathrm{Volt}$ bei $0^{\circ}$, beziehungsweise $21^{\circ}$ anlangt, so haben wir schon darauf verwiesen, daß ihre Lage von der An- oder Abwesenheit von $\mathrm{PbCl}_{2}$ in der Lösung ganz unabhängig ist. Welchem Elektrodenvorgang diese Knicke entsprechen, läßt sich einwandfrei nicht entscheiden.

Es ist bekannt, daß sich an Kohlenanoden cet. paribus mehr Sauerstoff entwickelt als an Platinelektroden. Es wäre nun denkbar, daß diesen ersten Knicken eine intermediäre Sauerstoffabscheidung entspricht, die durch die in hoher Konzentration vorhandenen Chlorionen depolarisiert wird, oder es sich um einen Oxydationsvorgang unter Mitwirkung des Elektrodenmaterials selbst handelt.

1 Abbeg's Handbuch der anorg. Chemie.

2 Zeitschr. für anorg. Chemie, 26, 162, 1903. 
Nach Joost ${ }^{1}$ und Nobis ${ }^{2}$ erhält man an Holzkohle in 2 norm. $\mathrm{H}_{2} \mathrm{SO}_{4}$ sich langsam einstellende Potentiale, die bis $\mathrm{zu}$ 0.75 Volt steigen, an sehr reinem Graphit in 2 norm. $\mathrm{H}_{2} \mathrm{SO}_{4}$ bei längerer Sauerstoffentwicklung ein Potential von 1.6 Volt, das allmählich auf niedere Werte (bis $+1 \cdot 1$ Volt) sinkt.

Bei der Zersetzung von 1 norm. $\mathrm{H}_{2} \mathrm{SO}_{4}$ an der von uns zu den früheren Versuchen verwendeten Bogenlampenkohle erhält man, wie aus den Versuchen 7 der Tabelle und der graphischen Darstellung in Fig. $2 \mathrm{zu}$ ersehen ist, einen Zersetzungspunkt bei 1.08 Volt, einen Wert, der der Sauerstoffelektrode entspricht. Wenn wir für die ersten Knickpunkte in Salzsäure die gleichen Elektrodenvorgänge annehmen, so liegt hier dieser Punkt um zirka 0.2 Volt niedriger.

Wie dem immer auch sei, werden aber bei den hohen Stromdichten bei der oben beschriebenen elektrochemischen Darstellung von Plumbichlorid (4 Amp. pro $d m^{2}$ auf die Kohlenanode) die den ersten Knickpunkten entsprechenden Vorgänge gegenüber den den zweiten Knickpunkten entsprechenden Vorgängen der Abscheidung von Chlorionen, für den die größte Ionenkonzentration zur Verfügung steht, in den Hintergrund treten, so daß es praktisch nur zur Abscheidung von Chlor kommt, beziehungsweise die Elektrode unangreifbar erscheint.

Wie bereits betont, geht aber aus unseren Versuchen hervor, daß bei der Elbs-Nübling'schen Darstellungsmethode von Plumbichlorid primär an den Anoden lediglich $\mathrm{PbCl}_{2}$ und $\mathrm{Cl}_{2}$ entstehen, die sekundär miteinander reagieren, ohne daß eine allfällige depolarisierende Wirkung des gelösten $\mathrm{PbCl}_{2}$ auf die Chlorabscheidung eine Rolle spielt.

1 Diss. Dresden 1910, p. 39.

2 Diss. Dresden 1909, p. 91. 